\title{
Análise dos impactos biopsicossociais da hanseníase em adultos
}

\author{
Analysis of biopsycosocial impacts of leprosy in adults \\ Análise de los impactos biopsicosociales de la lepra em adultos
}

Vitória dos Santos Cruz

ORCID: https://orcid.org/0000-0002-6773-7424

Faculdade Pitágoras Teixeira de Freitas, Brasil

E-mail: vitoria0snt@gmail.com

Yesly Marinho da Rocha Barreto

ORCID: https://orcid.org/0000-0002-5610-1420

Centro Universitário Brasileiro, Brasil

E-mail: marinhoyesly@gmail.com

Hozana Fernanda da Silva

ORCID: https://orcid.org/0000-0002-7210-4859

Universidade Maurício de Nassau, Brasil

E-mail: hznfernanda@gmail.com

Othávio Cardoso de Oliveira

ORCID: https://orcid.org/0000-0002-9401-4969

Universidade Salgado de Oliveira, Brasil

E-mail: o.cardoso.1998@gmail.com

Luana Rocha Lopes

ORCID: https://orcid.org/0000-0002-3268-5628 Centro Universitário Brasileiro, Brasil

E-mail: lualopess22@gmail.com

Lucas Sousa Penha

ORCID: https://orcid.org/0000-0002-6634-1081

Faculdade Santa Terezinha, Brasil

E-mail: lucassousasales2014@gmail.com

Guilherme Henrique Santana

ORCID: https://orcid.org/0000-0002-1897-2719

Centro Universitário Estácio do Recife, Brasil

E-mail: guilhermesanttanaenf@gmail.com

Claudia Danielle Guimarães

ORCID: https://orcid.org/0000-0002-2486-903X

Faculdade Integrada Cete, Brasil

E-mail: dani.36bernardo@gmail.com

Marília Dutra Teixeira

ORCID: https://orcid.org/0000-0003-2706-2810

Centro Universitário de Viçosa, Brasil

E-mail: mariliadutra04@gmail.com

Vitoria Vilas Boas da Silva Bomfim

ORCID: https://orcid.org/0000-0003-4897-0279

Centro Universitário Jorge Amado, Brasil

E-mail: pesquisaclinica9@gmail.com

Emily da Silva Eberhardt

ORCID: https://orcid.org/0000-0003-2736-5686

Centro Universitário Cenecista de Osório, Brasil

E-mail: milyeberhardt@gmail.com

Brenda Tayrine Tavares Souza

ORCID: https://orcid.org/0000-0003-4469-3198

Centro Universitário do Distrito Federal, Brasil

E-mail: brenndatayrine@gmail.com

Beatriz Kelly Oliveira Alves

ORCID: https://orcid.org/0000-0001-6318-9304

Universidade Salvador, Brasil

E-mail: beatrizallves102030@hotmail.com

Gleice Kelly Calixto Solidade Marques

ORCID: https://orcid.org/0000-0001-8977-2733

Centro Universitário Anhanguera, Brasil

E-mail: gleicekellymarques@gmail.com

Maria Rosemary da Silva Gomes

ORCID: https://orcid.org/0000-0002-8217-3647

Centro Universitário Santo Agostinho, Brasil

E-mail: mariarosemary2010@gmail.com 


\begin{abstract}
Resumo
Objetivo: Descrever o impacto da hanseníase na qualidade de vida dos portadores. Métodos: Trata-se de uma revisão bibliográfica integrativa realizada nas bases de dados Scientific Electronic Library Online (SciELO) e Banco de Dados em Enfermagem (BDENF). Como critérios de inclusão: artigos que abordem a temática, nos últimos dez anos. Como critérios de exclusão: artigos que não contemplavam o tema. Foram encontrados 85 estudos nas bases selecionadas e após aplicar os critérios de inclusão e exclusão, foram selecionados 11 estudos para compor a revisão. Resultados: A hanseníase é uma doença transmissível, que afeta os nervos periféricos e a pele, podendo causar sérios prejuízos sensoriais, motores e autônomos. É decorrente da suscetibilidade dos indivíduos, predisposição genética ou contato prolongado com portadores da hanseníase sem tratamento. Sabe-se que a hanseníase atualmente tem cura e seu tratamento é ofertado através do Sistema Único de Saúde (SUS), no entanto fatores como o estigma, preconceito e a falta de informação retardam o processo de cura da doença e traz consequências desafiadoras no âmbito da saúde pública. Considerações Finais: Portanto, torna-se pertinente à discussão do problema, com intuito de identificar estratégias que melhor minimizem o impacto da hanseníase na vida de portadores e os efeitos colaterais para coletividade.
\end{abstract}

Palavras-chave: Hanseníase; Saúde mental; Adultos.

\begin{abstract}
Objective: To describe the impact of leprosy on the quality of life of patients. Methods: This is an integrative literature review carried out in the Scientific Electronic Library Online (SciELO) and Nursing Database (BDENF) databases. As inclusion criteria: articles that address the theme, in the last ten years. As exclusion criteria: articles that did not cover the topic. Eighty-five studies were found in the selected databases and after applying the inclusion and exclusion criteria, 11 studies were selected to compose the review. Results: Leprosy is a transmissible disease that affects the peripheral nerves and the skin, and can cause serious sensory, motor and autonomic damage. It results from the individuals' susceptibility, genetic predisposition or prolonged contact with untreated leprosy patients. It is known that leprosy is currently curable and its treatment is offered through the Unified Health System (SUS), however factors such as stigma, prejudice and lack of information delay the process of curing the disease and bring challenging consequences in the context of public health. Final Considerations: Therefore, it is pertinent to discuss the problem, in order to identify strategies that better minimize the impact of leprosy on the lives of patients and the side effects for the community.
\end{abstract}

Keywords: Leprosy; Mental health; Adults.

\begin{abstract}
Resumen
Objetivo: Describir el impacto de la lepra en la calidad de vida de los pacientes. Métodos: Se trata de una revisión integradora de la literatura realizada en las bases de datos Scientific Electronic Library Online (SciELO) y Nursing Database (BDENF). Como criterio de inclusión: artículos que aborden el tema, en los últimos diez años. Como criterio de exclusión: artículos que no cubrieron el tema. Se encontraron 85 estudios en las bases de datos seleccionadas y luego de aplicar los criterios de inclusión y exclusión, se seleccionaron 11 estudios para componer la revisión. Resultados: La lepra es una enfermedad transmisible que afecta los nervios periféricos y la piel, pudiendo causar graves daños sensoriales, motores y autonómicos. Es el resultado de la susceptibilidad, la predisposición genética o el contacto prolongado de los individuos con pacientes con lepra no tratados. Se sabe que la lepra actualmente es curable y su tratamiento se ofrece a través del Sistema Único de Salud (SUS), sin embargo factores como el estigma, el prejuicio y la falta de información retrasan el proceso de curación de la enfermedad y traen consecuencias desafiantes en el contexto de la salud pública. Consideraciones finales: Por lo tanto, es pertinente discutir el problema, con el fin de identificar estrategias que minimicen mejor el impacto de la lepra en la vida de los pacientes y los efectos secundarios para la comunidad.
\end{abstract}

Palabras clave: Lepra; Salud mental; Adultos.

\title{
1. Introdução
}

A hanseníase constitui um sério problema de saúde pública pelo seu impacto coletivo e individual, embora a medicina tenha avançado no tratamento da doença, culturalmente a hanseníase ainda é estigmatizada na sociedade brasileira. Os preconceitos sofridos pelos portadores de tal são indubitavelmente relevantes para considerar vários fatores que predispõem o agravamento desta doença, como não adesão ao tratamento e impacto psicossocial da estigmatização (Palmeira et al., 2020).

Conceitualmente a hanseníase é uma das doenças mais antigas na história da humanidade e, pode ser definida como sendo uma doença transmissível causada pelo Mycobacterium lepra e através da suscetibilidade dos indivíduos, predisposição genética ou contato íntimo e prolongado com portadores da hanseníase sem tratamento, habitação entre outras. Essa doença afeta principalmente os nervos periféricos e a pele, podendo o seu agravamento causar sérios prejuízos sensoriais, motores e 
autônomos (van‘t Noordende et al., 2020). A hanseníase aparece em evidência sendo entretanto, altas taxas de novos casos são notificadas em todo o mundo a cada ano. No Brasil em 2015 houve a notificação de mais de 28.000 mil novos casos (Silva $e t$ al., 2019).

O diagnóstico e tratamento precoce da hanseníase são necessários para minimizar os impactos da mesma, como as incapacidades e deformidades nos nervos periféricos e em estágio mais avançado o acometimento neurológico. As limitações físicas que a hanseníase pode trazer atrapalha as relações interpessoais e reflete na qualidade de vida do portador. Além de incapacidades físicas, merece atenção às repercussões de caráter emocional da hanseníase com consequências de morbidade da enfermidade devendo ser pauta no tratamento e controle da doença (Silva et al., 2019).

Entende-se que a descentralização do atendimento nos centros de referência para as Unidades Básicas de saúde, é um importante método auxiliador no enfrentamento à doença, pois o mesmo promove o acesso facilitado ao tratamento através do vínculo estabelecido pela equipe multiprofissional e comunidade assistida e minimiza as barreiras físicas e sociais e impedem o tratamento de muitos doentes. No entanto, apenas isso não diminui o acometimento da doença, precisando as políticas de saúde serem voltadas a essa temática. Ademais, este estudo evidencia que o Nordeste apresenta menores de 14 anos acometidos pela doença com incapacidades físicas, mostrando a proporção da doença no país (Silva et al., 2020).

Segundo Palmeira et al. (2020) a hanseníase causa desequilíbrios que rompem a homeostasia das necessidades humanas básicas descritas por Maslow, o resguardo por medo de represálias e preconceitos que podem estar presentes no trabalho, nos círculos de amizades e até no lugar onde todo indivíduo deveria se sentir seguro e entendido, o próprio lar, faz com que portadores de hanseníase negligenciam a própria saúde e retarda o tratamento.

Estudos alertam a ligação que determinantes sociais de saúde tem para a infecção e agravamento da hanseníase. Todo o contexto de vida que o indivíduo está inserido é relevante, como sociopolítico, sociodemográfico e socioeconômico e pode implicar no pior prognóstico da enfermidade. Outro fator cuja informação permanece parcial é a variedade de hospedeiros de M. leprae, o mesmo já foi identificado em solo, água e até esquilos, o que favorece a disseminação da doença e está inteiramente ligado à habitação do indivíduo. A Organização Mundial da Saúde (OMS) identificou que a maior parte de novas infecções foram evidenciadas nos países de prioridade global, o que contribui para essa tese, todavia, a confirmação permanece parcial (Araújo et al., 2020).

A hanseníase constitui um sério problema de saúde pública pelo seu impacto coletivo e individual, já que estamos falando também de um estigma. Os preconceitos sofridos pelos portadores de tal são indubitavelmente relevantes para considerar vários fatores que predispõem o agravamento desta mazela, como não adesão ao tratamento e impacto psicossocial da estigmatização (Palmeira et al., 2020).

Faz-se, portanto, essencial a educação em saúde para essa população, embasada nos aspectos clínicos fundamentais para o sucesso do tratamento, mas também em aspectos subjetivos da doença. Pelo supracitado, objetiva-se com este estudo, identificar o impacto da hanseníase em todo o contexto de vida de portadores adultos, e estabelecer subsídios que promova qualidade de vida, como informações fidedignas e a disseminação da desmistificação do estigma que é uma construção social e sobretudo, mutável.

\section{Metodologia}

Trata-se de uma revisão bibliográfica integrativa que se define como uma pesquisa desenvolvida através de materiais já elaborados, principalmente, artigos científicos, fornecendo uma visão mais ampla sobre determinado assunto, sendo esta, conduzida por uma questão de pesquisa construída de maneira clara e objetiva (Ercole et al., 2014). O estudo sustentou-se a partir da seguinte questão norteadora: "Quais impactos a hanseníase trás para seus portadores nos âmbitos biológico, psicológico e social?". O levantamento dos artigos realizou-se entre os meses de agosto e novembro de 2020, através da 
Biblioteca Virtual em Saúde (BVS) com as bases de dados Literatura Latino-Americana e do Caribe em Ciências da Saúde (LILACS), Scientific Electronic Library Online (SciELO), Medical Literature Analysis and Retrieval System Online (MEDLINE), Pubmed e também Revista Acervo Saúde. A partir dos Descritores em Ciências Saúde (DeCS): Hanseníase, saúde mental, adulto. Os descritores foram cruzados através do operador booleano "AND” para busca simultânea dos assuntos.

Foram considerados os seguintes critérios de inclusão: artigos nos idiomas português, inglês, com texto completo, publicados entre os anos de 2011 e 2021, disponível e com abordagem relacionada à temática do proposto neste estudo. E, como critérios de exclusão, artigos repetidos nas bases de dados, artigos de revisão, teses, dissertações e capítulos de livro.

A partir dos critérios estabelecidos, foram encontrados 85 estudos. Após leitura pareada dos títulos e resumos para constatar quais se adequaram aos critérios de inclusão e exclusão estabelecidos, 11 estudos foram excluídos por não se tratarem de artigos, 16 foi excluído por não estar disponível na íntegra, 31 foram excluídos por não abordarem a temática do estudo e 12 foram excluídos por estarem repetidos nas bases de dados, sendo selecionados 15 estudos para compor a revisão, vide (Figura 1).

A análise de dados foi realizada por meio de um banco de dados montado no programa Excel versão 2016, no qual os mesmos foram convertidos e apresentados em forma de tabela, com o intuito de demonstrar os resultados obtidos atendendo aos objetivos da pesquisa. Tais resultados foram confrontados com as evidências disponíveis na literatura e discutidos com o embasamento científico. Por ter como referência bases públicas, não será preciso a submissão ao Comitê de Ética e Pesquisa, respeitando, porém, os preceitos éticos estabelecidos na resolução número 466/2012 do Conselho Nacional de Saúde (Brasil, 2012).

Figura 1 - Seleção da Amostra de Artigos, Brasil (2021).

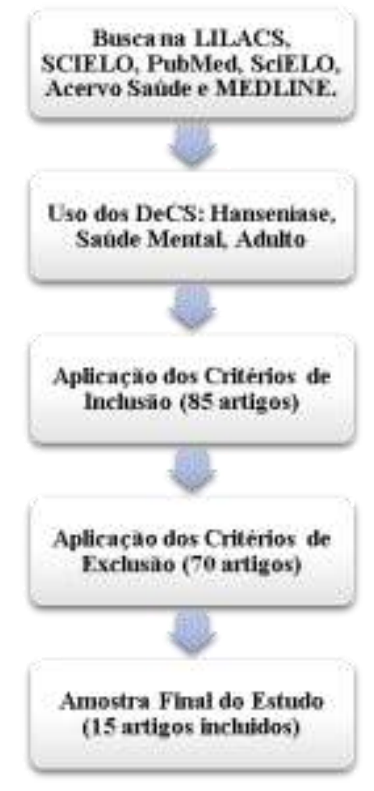

Fonte: Autores (2021).

\section{Resultados}

Os artigos encontrados nas bases de dados foram submetidos a uma criteriosa leitura analítica, crítica e eletiva, sendo aplicados os critérios de inclusão e exclusão, compondo esta revisão integrativa 21 artigos (Quadro 1).

Dos 21 artigos selecionados, 16 foram publicados em periódicos brasileiros, 5 são referências internacionais. Os estudos foram publicados entre 2011 e 2021, sendo a maioria publicada em 2020. Todos os estudos estão em consonância com o objetivo a ser abordado no estudo. 
Quadro 1 - Síntese dos principais achados sobre o Impacto Biopsicossocial da Hanseníase em Adultos, 2021.

\begin{tabular}{|c|c|c|}
\hline $\mathbf{N}$ & Autores (Ano) & Principais achados \\
\hline 1 & da Silva et al. (2020) & $\begin{array}{l}\text { Trata-se de uma pesquisa epidemiológica de caráter descritivo e abordagem quantitativa, } \\
\text { utilizando dados provenientes do Sistema de informação de agravos de notificação } \\
\text { (SINAN). Analisando o perfil clínico-epidemiológico de pacientes portadores de } \\
\text { hanseníase em um município maranhense no período de } 2015 \text { a } 2017 \text {. }\end{array}$ \\
\hline 2 & $\begin{array}{l}\text { Kabir \& Hossain } \\
\text { (2019) }\end{array}$ & $\begin{array}{l}\text { Amostragem Intencional, questionário estruturado pré-testado. Este estudo demonstrou } \\
\text { que o treinamento melhorou o conhecimento entre todos os quadros de provedores de } \\
\text { serviços, embora variou entre aqueles que prestaram serviços diretos e aqueles que estão } \\
\text { parcialmente. }\end{array}$ \\
\hline 3 & Xiong et al. (2019) & $\begin{array}{l}\text { Pesquisa transversal, questionário geral de saúde e questionário de qualidade de vida da } \\
\text { Organização Mundial da Saúde. Conclui-se que além de focar nos fatores associados à má } \\
\text { qualidade de vida e saúde psicológica entre pessoas afetadas pela Hanseníase, há uma } \\
\text { necessidade urgente de redução do estigma, programas de reabilitação e integração social. } \\
\text { Isso pode ser alcançado envolvendo os membros da comunidade junto com as pessoas } \\
\text { afetadas pela Hanseníase para projetar um programa de intervenção adaptado localmente. }\end{array}$ \\
\hline 4 & Cunha et al. (2019) & $\begin{array}{l}\text { Análise de dados epidemiológicos da prevalência de hanseníase do Sistema de Informação } \\
\text { de Agravos de Notificação (SINAN) no período de } 2014 \text { a } 2017 \text {. A faixa etária mais } \\
\text { acometida é a população de até } 40 \text { anos. Foram registrados } 224 \text { casos de hanseníase, } \\
\text { observou-se que mais da metade dos pacientes obtiveram cura. }\end{array}$ \\
\hline 5 & $\begin{array}{l}\text { Marahatta et al. } \\
\text { (2018) }\end{array}$ & $\begin{array}{l}\text { Estudo de caso quantitativo, investigação da cultura da população. Constata-se que, a } \\
\text { discriminação que o estigma gerado através do preconceito traz é significativa e afeta a } \\
\text { vida de portadores em âmbitos físicos, de interação social e impacta a saúde psíquica, } \\
\text { sendo necessária a discussão sobre tal problema. }\end{array}$ \\
\hline 6 & $\begin{array}{l}\text { Govindharaj et al. } \\
\text { (2018) }\end{array}$ & $\begin{array}{l}\text { Estudo transversal descritivo, Questionário semiestruturado. Portanto, este estudo tenta } \\
\text { explorar a gravidade da doença percebida por pessoas afetadas pela hanseníase que } \\
\text { notificam no centro de referência para hanseníase, Purulia, West Bengal, Índia. }\end{array}$ \\
\hline 7 & Pryce et al. (2018) & $\begin{array}{l}\text { Estudo de caso qualitativo, participação social. Percebe-se a necessidade de incentivar o } \\
\text { autocuidado na população portadora de hanseníase e informar acerca do acesso aos } \\
\text { serviços de saúde. }\end{array}$ \\
\hline 8 & Reis et al. (2017) & $\begin{array}{l}\text { Estudo transversal, questionário de múltipla escolha. Pode-se concluir que, para a amostra } \\
\text { estudada, não foi observada associação entre as limitações de atividades, avaliadas pela } \\
\text { escala Salsa, nem a restrição de participação social, avaliada pela Escala de Participação, } \\
\text { com os dados demográficos analisados. }\end{array}$ \\
\hline 9 & $\begin{array}{l}\text { Gonçalves et al. } \\
\text { (2018) }\end{array}$ & $\begin{array}{l}\text { Estudo de caso com mulheres na convivência com a hanseníase, participação social. Ficou } \\
\text { apercebido o impacto da hanseníase na vida de portadoras mulheres, trazendo empecilhos } \\
\text { para realização de atividades pessoais como cuidado com a família e vida profissional, o } \\
\text { que alarga a desigualdade social sendo um problema pertinente de saúde pública }\end{array}$ \\
\hline 10 & $\begin{array}{l}\text { Quaresma et al. } \\
\text { (2019) }\end{array}$ & $\begin{array}{l}\text { Este estudo identificou um predomínio de portadores da hanseníase no gênero masculino e } \\
\text { adultos. Na população estudada foram identificados poucos casos de incapacitação o que } \\
\text { leva os infectados ao tratamento na Atenção Primária à Saúde. }\end{array}$ \\
\hline 11 & $\begin{array}{l}\text { de Macedo et al. } \\
\text { (2019) }\end{array}$ & $\begin{array}{l}\text { A estimativa de tendência para } 2020 \text { demonstrou que o Brasil manterá o índice em } \\
\text { redução lenta e gradativa, enquanto o estado do Pará seguirá com índices elevados, a } \\
\text { incidência de casos multibacilares são maiores, o estudo sugere uma a necessidade de } \\
\text { aperfeiçoar o monitoramento }\end{array}$ \\
\hline 12 & Garbin et al. (2015) & $\begin{array}{l}\text { Este estudo transversal descritivo com abordagem quantitativa buscou saber o nível de } \\
\text { conhecimento acerca da doença e qualidade de vida de pacientes hansenianos que } \\
\text { realizam tratamento nas Unidades de Saúde de Cuiabá, no Mato Grosso. Foi aplicado um } \\
\text { questionário durante as consultas que estava dividido em categorias, como: dados } \\
\text { sociodemográficos, conhecimento da doença, participação social, estigma, auto-estima e } \\
\text { qualidade de vida. O estudo revelou que dos } 94 \text { pacientes, } 63 \text { afirmaram estar deprimidos } \\
\text { após o diagnóstico. Além disso, } 63,3 \% \text { afirmaram ter medo de rejeição. }\end{array}$ \\
\hline
\end{tabular}




\begin{tabular}{|c|c|c|}
\hline 13 & $\begin{array}{l}\text { Cavalcante } \text { et al. } \\
\text { (2020) }\end{array}$ & $\begin{array}{l}\text { As deficiências na detecção precoce da hanseníase foi um ponto importante apontado } \\
\text { neste estudo, evidenciado através da identificação de casos multibacilares e ainda graus de } \\
\text { incapacidades. }\end{array}$ \\
\hline 14 & Silva et al. (2020) & $\begin{array}{l}\text { Os resultados deste estudo evidenciaram que mais uma vez a deficiência a detecção } \\
\text { precoce é uma das causas primordiais que podem causar agravamento da doença podendo } \\
\text { levar a incapacitação. }\end{array}$ \\
\hline 15 & Garcia et al. (2020) & $\begin{array}{l}\text { Foi identificado neste estudo que a vacina BCG tem papel fundamental na prevenção da } \\
\text { hanseníase, sendo a primeira dose fundamental para prevenir os casos multibacilares em } \\
\text { mulheres e a segunda dose a forma clínica da doença em homens. }\end{array}$ \\
\hline
\end{tabular}

Fonte: Autores (2021).

O estudo de número 1 identifica que o maior número de diagnósticos da hanseníase em adultos é feito de forma espontânea e que a maior parte das pessoas que saíram do tratamento acompanhadas neste estudo, foram pessoas curadas. No entanto, ações de combate à doença na região devem ser continuamente implementadas (da Silva et al., 2020). O estudo de número 4 evidencia dados que complementam artigos que serão citados, como o predomínio da hanseníase na população masculina, maioria chefe de família economicamente ativa (Cunha et al., 2019).

O artigo 3 caracteriza a população do estudo, onde mais de $91 \%$ tinham mais de 50 anos, e aproximadamente $80 \%$ tinham apenas o ensino fundamental completo e mais de $70 \%$ estavam em situação de desemprego. A maior parte dos entrevistados relataram problemas de cunho psicológico (Xiong et al., 2019). Já o estudo 6 identificou que 60\% dos estudados eram homens, com até 45 anos, 55\% eram trabalhadores braçais com renda familiar mensal menor que 5000 mil reais (Govindharaj et al., 2018).

O artigo 6 identifica que mais da metade da população da pesquisa possui deficiência física (Govindharaj et al., 2018). $\mathrm{O}$ artigo de número 8 indica que a maior porcentagem de pessoas com incapacidades físicas não concluiu o ensino médio (Reis, Castro \& Fernandes, 2017).

Portadores de hanseníase devem estar cientes da importância do autocuidado, segundo Pryce et al. (2018) no artigo de número 7 aborda que aproximadamente $92 \%$ dos abordados tinham ciência do manejo necessário à sua condição clínica, no entanto, apenas $21 \%$ praticavam os métodos de autocuidado preconizados.

A percepção dos sentimentos e emoções vivenciados pelos portadores de hanseníase descrito no artigo 6 são em sua maioria de medo, vergonha, tristeza e ansiedade (Govindharaj et al., 2018). O estudo de número 5 também aborda o impacto psicológico que a hanseníase traz, o estigma que a doença acarreta leva ao ocultamento da mesma por parte dos portadores. Ademais, o impacto físico que promove nos casos mais graves o acometimento de deformidades, além do impacto na vida econômica dos mesmos, pois muitos descrevem a deformidade como impedimento para conseguir emprego (Marahatta et al., 2018).

Podemos observar no estudo 9 a presença da dor física em muitos relatos, além do sofrimento que o diagnóstico traz, a incapacidade de trabalhar gera diminuição na renda familiar, algumas atividades do cotidiano ficam impossibilitadas aumentando a angústia e preocupação (Gonçalves et al., 2018).

$\mathrm{O}$ artigo de número 2 evidenciou que nem todos os profissionais estavam preparados para identificar e lidar com complicações da hanseníase. Demonstrando diminuição no conhecimento da abordagem como aconselhamento e investigação laboratorial. No entanto, após implementação do projeto de aperfeiçoamento dos profissionais houve melhora significativa (Kabir \& Hossain, 2019). 
No estudo 10 é apercebido que o Brasil se encontra entre um dos países com grande índice de infecção, isso deve-se também pelo país ter grande extensão territorial (Quaresma et al., 2019). O estudo 11 afirma ainda que a distribuição e a evolução da hanseníase para ser avaliada deve levar em consideração, aspectos clínicos, demográficos e também territoriais (de Macedo et al., 2019).

O estudo 12 revelou que o paciente que sofre de hanseníase possui um olhar preconceituoso de si mesmo e das outras pessoas, podendo esse comportamento levá-lo a ocultar a doença. Tal fato contribui de forma significativa para o isolamento social do doente pelo medo de rejeição. (Garbin et al., 2015).

Nos estudos 13 e 14 foram avaliados fatores que predispõe o agravamento da hanseníase e evidenciado que a falta do diagnóstico precoce é um fator determinante, sendo proveniente o acometimento agressivo da enfermidade e consequentemente diversos graus de incapacidades físicas, atingindo principalmente populações menos favorecidas e áreas historicamente endêmicas (Cavalcante et al., 2020).

As evidências trazidas pelo estudo 15 são importantíssimas e passíveis de disseminação. Trata-se da relação da vacina BCG com a prevenção da hanseníase (Garcia et al., 2020), uma doença cuja o diagnóstico precoce favorece a minimização dos agravos, a prevenção através da vacina cientificamente atestada é uma ferramenta cujo uso é necessário e as consequências positivas indubitavelmente reais.

\section{Discussão}

A hanseníase atinge principalmente populações menos favorecidas, como pessoas com grau de escolaridade baixo, baixa renda, com emprego informal, residentes em áreas que são historicamente endêmicas, reflexo e consequências das desigualdades sociais. Algumas estratégias de saúde precisam ser continuadas no que diz respeito ao diagnóstico e tratamento precoce da hanseníase, este estudo evidenciou que a vigilância de contatos intradomiciliares é uma estratégia excelente e por isso, deve ser vastamente utilizada nos serviços de saúde, que por vezes negligencia esse problema de saúde pública. Ademais, estratégias de prevenção devem ser as de primeira escolha, enfatizando o estigma, que na maioria das vezes é perpetuado pelos próprios portadores da doença e é um dos principais causadores de não adesão e/ou abandono do tratamento (Cavalcante et al., 2020).

Muitos indivíduos tinham em média 65 anos, eram portadores de doenças crônicas, e o nível de escolaridade era baixo, o que somado à hanseníase propiciava problemas psicológicos, estando mais suscetíveis idosos e mulheres. As pessoas não relacionam saúde mental à qualidade de vida, sendo um problema subestimado (Xiong et al., 2019).

Por conta do medo da discriminação muitos indivíduos acometidos pela hanseníase, optam por não partilhar com o ciclo social sua condição de doença, o que causa preocupação por se tratar de uma infecção transmissível. Ademais, as deformidades e incapacitações trazidas pela enfermidade impactam a economia dos indivíduos, os colocando em situação de vulnerabilidade para surgimento de problemas psicológicos que como supracitado acima, é subestimado, mas tem um impacto significativo na vida dos enfermos, seus contos próximos e ainda reflete na coletividade em diversos contextos, como na economia dos sistemas públicos de saúde (Marahatta et al., 2018).

As mudanças nas relações sociais são perceptíveis, a vergonha leva ao isolamento, isso traz sofrimento psíquico, pois impede o cuidado e atenção dos familiares e amigos que formam a rede de apoio. A dor física é somada a dor social, além das incapacidades e deformidades, a discriminação é um fator condicionante para o adoecimento (Gonçalves et al., 2018)

Atualmente a hanseníase tem cura e a disseminação do conhecimento acerca de seu tratamento é maior. No entanto, o preconceito e estigma vivenciado por portadores, aumenta a sensibilidade dos sentimentos e emoções negativos, como a ansiedade, a tristeza e o medo. O risco de incapacitações físicas e deformidades geram receios de convivência social e baixa 
autoestima. O diagnóstico precoce diminui a intensidade dessas emoções e também o risco de complicações físicas e doenças emocionais (Govindharaj et al., 2018).

Em uma pesquisa Pryce et al. (2018) identifica a crescente positiva no conhecimento de portadores de hanseníase acerca do autocuidado e sua significativa na melhora do quadro clínico, no entanto é possível notar alguns fatores que interferem nas práticas de autocuidado como condição econômica, que impossibilita a compra de cremes e sabões. Esses fatores necessitam de atenção pois não permitem o cuidado integral e promovem risco de avanço e complicações da doença. A baixa escolaridade é citada em todos os estudos desta pesquisa e compreende um fator de risco para a hanseníase, estudos corroboram que pessoas com nível de escolaridade inferior não aderem ao tratamento de forma disciplinar, por desconhecerem as informações que resultam em entendimento do problema a ser enfrentado e vencido (Reis et al., 2017).

É importante ressaltar, que conhecer o perfil dos portadores de hanseníase auxilia no diagnóstico das principais dificuldades enfrentadas pelos mesmos, abrindo espaços para debates que lancem estratégias que minimizem os impactos biopsicossociais da hanseníase, como promoção da autoestima, do autocuidado e reinserção social (Reis et al., 2017).

Segundo Kabir e Hossain (2019) é essencial aos serviços de saúde que os profissionais sejam capacitados para diagnosticar precocemente casos de hanseníase, o diagnóstico precoce favorece o tratamento precoce, isso impede o surgimento de complicações que causam incapacidades. É relevante dizer que o diagnóstico da hanseníase pode ser feito com anamnese e exame físico, muitas vezes os diagnósticos provenientes de tecnologias leves são subestimados, no entanto, a valorização dos mesmos sustenta o cuidado eficiente (Gonçalves et al., 2018).

Atualmente, os estudos identificam flutuações nas taxas de incidência da hanseníase, isso se dá também pelo pressuposto que controlar a doença é ainda uma dificuldade. Isso reflete mais uma vez a necessidade de incentivar a adesão ao tratamento, um estudo indicou que aproximadamente $99,5 \%$ da população do estudo tinham aderido ao tratamento, e $90 \%$ eram provenientes de encaminhamentos das unidades de Atenção Primária que prestam serviços de saúde, sendo estas reconhecidas como fundamentais na busca ativa de infectados, seus tratamentos e promoção da qualidade de vida (Quaresma $e t$ al., 2019).

A Atenção Básica tem potencial grandioso no diagnóstico precoce e combate à hanseníase, no entanto, algumas deficiências são identificadas como na descentralização da assistência à hanseníase, precisam ser sanadas para que seus recursos possam ser utilizados em sua máxima potência. O que pode ser utilizado como subsídio para estimular o uso dos serviços públicos de saúde, são programas sociais que estimulam o aumento a frequência escolar e busca meios de promover o sustento às famílias de extrema pobreza e outras, refletindo tais ações nos indicadores de saúde das pessoas mais suscetíveis (Cavalcante et al., 2020).

Segundo Silva et al. (2020)., observou-se com base na população de estudo que as características eram semelhantes às divulgadas pelo boletim epidemiológico nacional, sendo o aumento de casos multibacilares principalmente, e diagnósticos em menores de 15 anos, sugestivo de transmissão ativa, constatando a literatura mais uma vez que as estratégias de controle da doença devem ser aperfeiçoadas, e ainda que as estratégias de prevenção devem ser amplamente estimuladas, como o esquema vacinal da BCG completo.

Existem diversos fatores que explicam o aumento de casos de hanseníase no Brasil, seja os fatores de vulnerabilidades das populações ou aqueles de transformação na modalidade de vida, como a inserção das mulheres no mercado de trabalho, e até mesmo negligências dos sistemas de saúde com determinadas populações, por tanto, neste estudo epidemiológico, evidências fidedigna da funcionalidade da vacina BCG na prevenção da hanseníase em mulheres e homens, que se classifica como instrumento potencialmente efetivo e necessário no combate a problemática (Garcia et al., 2020). 


\section{Considerações Finais}

Pelo já supracitado, percebe-se a grande necessidade de discutir sobre o impacto individual e coletivo da hanseníase. Essa enfermidade altera o organismo do indivíduo portador, promovendo transformações físicas, além da manifestação de dor e desconforto. Acarreta estigma e preconceito, alterando ainda o ciclo social com o afastamento do trabalho, convívio com amigos e familiares e impacta a coletividade com geração de custos ao Sistema Único de Saúde por exemplo. Esse problema de saúde pública merece atenção direcionada para sua resolução. O diagnóstico preventivo de riscos para as populações vulneráveis, somado a uma eficiente educação em saúde, são estratégias preventivas primordiais a serem implementadas nos serviços de Atenção Primária à Saúde. A assistência deve ser integral, multidisciplinar e contínua, tratando não apenas as questões fisiológicas da doença em si, mas os condicionantes que interferem no processo de tratamento e cura do indivíduo, como bem-estar social, psicológico, portanto, a capacitação dos profissionais de saúde é essencial. Ademais, a atenção à saúde deve ser ofertada aos familiares de portadores de hanseníase, que também estão vulneráveis a adoecimento psíquico, já que vivenciam todo processo patológico diretamente. Sugere-se ainda, o aprofundamento de novos estudos sobre a temática, com intuito de construir subsídios para serem usados na prática assistencial dos profissionais de saúde, como protocolos e fluxogramas do manejo clínico da hanseníase. E materiais de cunho educacional que elucidem as informações acerca da doença e derrube conhecimentos empíricos e pejorativos.

\section{Referências}

Araújo, O. D. D., Ferreira, A. F., Araújo, T. M. E. D., Silva, L. C. L. D., Lopes, W. M. P. S., Neri, É. A. R., \& Ramos Jr, A. N. (2020). Mortalidade relacionada à hanseníase no Estado do Piauí, Brasil: tendências temporais e padrões espaciais, 2000-2015. Cadernos de Saúde Pública, 36 , e00093919.

Cavalcante, M. D. M. A., Larocca, L. M., \& Chaves, M. M. N. (2020). Múltiplas dimensões da gestão do cuidado à hanseníase e os desafios para a eliminação. Revista da Escola de Enfermagem da USP, 54.

Cunha, D. V., Rodrigues, E. B., Lameira, H. A., da Cruz, M. T. S., Rodrigues, S. M., \& dos Santos, F. D. S. (2019). Perfil Epidemiológico da Hanseníase no Município de Castanhal-Pará no período de 2014 a 2017. Revista Eletrônica Acervo Saúde, 11(15), e858-e858.

da Silva, P. S. R., Cunha, N. G. T., Oliveira, L. S., \& Santos, M. C. A. (2020). Perfil clínico-epidemiológico de pacientes portadores de hanseníase em um município do Maranhão. Revista Eletrônica Acervo Saúde, 12(8), e3468-e3468.

de Macedo, G. M. M., de Almeida Queiroz, M. F., da Silva, A. R., da Silva Pinheiro, B. V., Franco, M. C. A., \& Xavier, M. B. (2019). Spatial distribution and temporal evolution of leprosy in an area of an old colony in the state of Pará. Revista Eletrônica Acervo Saúde, 11(12), e582-e582.

Ercole, F. F., Melo, L. S. D., \& Alcoforado, C. L. G. C. (2014). Revisão integrativa versus revisão sistemática. Revista Mineira de Enfermagem, 18(1), 9-12.

Garbin, C. A. S., Garbin, A. J. Í, Carloni, M. E. O. G., Rovida, T. A. S., \& Martins, R. J. (2015). The stigma and prejudice of leprosy: influence on the human condition. Revista da Sociedade Brasileira de Medicina Tropical, 48(2), 194-201.

Garcia, I. R., Santos, A. M. R. T., Pereira, P. F., \& da Silva, E. D. A. M. (2020). Análise epidemiológica de casos novos de hanseníase em uma antiga colônia do Estado de Minas Gerais (2000-2016). Saúde e Pesquisa, 13(4), 871-877.

Gonçalves, M., Prado, M. A. R. D., Silva, S. S. D., Santos, K. D. S., Araujo, P. N. D., \& Fortuna, C. M. (2018). Trabalho e hanseníase: mulheres em suas dores, lutas e labutas. Revista brasileira de enfermagem, 71, 660-667.

Govindharaj, P., Srinivasan, S., \& Darlong, J. (2018). Perception toward the disease of the people affected by leprosy. International journal of mycobacteriology, 7(3), 247.

Kabir, H., \& Hossain, S. (2019). Conhecimento sobre hanseníase e sua gestão entre prestadores de serviços de saúde primários em dois distritos de Bangladesh. BMC health services research, 19 (1), 1-8.

Marahatta, S. B., Amatya, R., Adhikari, S., Giri, D., Lama, S., Kaehler, \& Adhikari, B. (2018). Perceived stigma of leprosy among community members and health care providers in Lalitpur district of Nepal: A qualitative study. PLoS One, 13(12), e0209676.

Ministério da Saúde. (2012). Conselho Nacional de Saúde. Resolução n 466, de 12 de dezembro de 2012. Aprova normas regulamentadoras de pesquisas envolvendo seres humanos. https://bit.ly/1mTMIS3

Palmeira, I. P., Moura, J. N., Epifane, S. G., Ferreira, A. M. R., \& Boulhosa, M. F. (2020). Percepção de pacientes com hanseníase sobre suas necessidades humanas básicas alteradas: indícios para o autocuidado. Rev. Pesqui.(Univ. Fed. Estado Rio J., Online), 324-329.

Pryce, J., Mableson, H. E., Choudhary, R., Pandey, B. D., Aley, D., Betts, H., \& Cross, H. (2018). Avaliação da viabilidade da integração do autocuidado para o linfedema filarial em grupos comunitários de autoajuda em hanseníase existentes no Nepal. BMC Public Health , 18 (1), 1-13. 
Research, Society and Development, v. 10, n. 7, e38610716872, 2021

(CC BY 4.0) | ISSN 2525-3409 | DOI: http://dx.doi.org/10.33448/rsd-v10i7.16872

Quaresma, M. D. S. M., Souza, L. D. S. C., da Silva, F. B. M., Pontes, C. D. N., \& da Silva, Y. J. A. (2019). Perfil clínico e epidemiológico dos pacientes portadores de hanseníase em uma unidade de referência no estado do Pará. Revista Eletrônica Acervo Saúde, (18), e269-e269.

Reis, B. M., Castro, S. S. D., \& Fernandes, L. F. R. M. (2017). Limitação de atividade e restrição de participação social em relação a faixa etária, gênero e escolaridade em pessoas com hanseníase. Anais brasileiros de dermatologia , 92 (3), 335-339.

Silva, C. R. D., Sampaio, L. H. F., Souza, J. P. F. D., Caetano, G. T. P., \& Wastoswki, I. J. (2020). Epidemiological trends of leprosy in Goiás, Brasil. Ciência \& Saúde Coletiva, 25, 3723-3730.

Silva, C. S. D., Barreto, J. A., Avelino-Silva, V. I., \& Bierrenbach, A. L. (2020). Impact of health interventions on epidemiological and operational leprosy indicators in a hyperendemic municipality of Brazil. Revista do Instituto de Medicina Tropical de São Paulo, 62.

Silva, P. M. F., Pereira, L. E., Ribeiro, L. L., dos Santos, D. C. M., do Nascimento, R. D., \& D'Azevedo, S. S. P. (2019). Evaluation of the physical limitations, psychosocial aspects and quality of life of people affected by leprosy/Avaliação das limitações físicas, aspectos psicossociais e qualidade de vida de pessoas atingidas pela hanseníase. Revista de Pesquisa: Cuidado é Fundamental Online, 11(1), 211-215.

van't Noordende, A. T., Aycheh, M. W., \& Schippers, A. (2020). The impact of leprosy, podoconiosis and lymphatic filariasis on family quality of life: a qualitative study in northwest Ethiopia. PLoS neglected tropical diseases, 14(3), e0008173.

Xiong, M., Wang, X., Su, T., Yang, B., Li, M., \& Zheng, D. (2019). Relationship between psychological health and quality of life of people affected by leprosy in the community in Guangdong province, China: a cross-sectional study. BMC public health, 19(1), 1-8. 